\title{
Congenital diaphragmatic hernia
}

\author{
Azyan Shafeek ${ }^{1}$, Stella G de Silva ${ }^{2}$, Ranjan Dias ${ }^{3}$
}

Sri Lanka Journal of Child Health, 2000; 29: 124

(Key words: Congenital diaphragmatic hernia)

A 3 month old female child presented with tachypnoea, incessant crying and restlessness at night. She was the first born, after 7 years of marriage, delivered by caesarean section at 37 weeks of gestation due to PIH:, birth weight was of $2.1 \mathrm{~kg}$.

She was tachypnoeic and the mother had to put her on her shoulder propped up to assist sleep. The child was accepting feeds well and was gaining weight normally (5.9 kg on admission).

On examination the child had a respiratory rate of $64 /$ minute. There was subcostal and intercostal recession but no cyanosis. There was reduced air entry on the right side, shifting of the heart to the left and a diagnosis of the right sided diaphragmatic

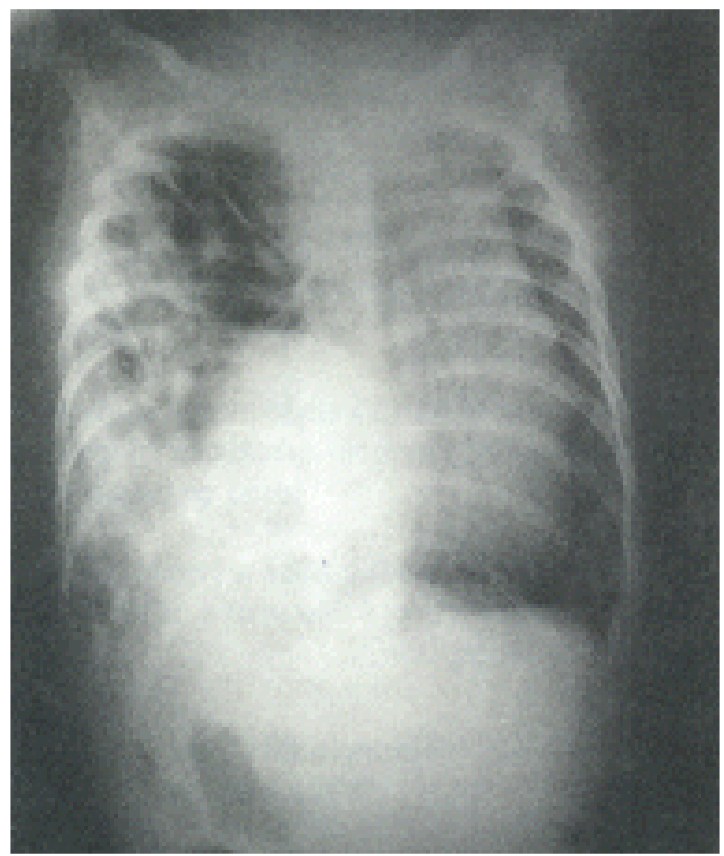

Figure 1 Right sided diaphragmatic hernia (Before surgery) hernia was made. The more common and more easily diagnosed left sided diaphragmatic hernia has a shift of the heart to the right causing more respiratory distress. The right sided diaphragmatic hernia was confirmed by chest X-ray (Figure 1). This shows the bowel loops and liver on the right side of the chest, shift of the mediastinum to the left and right upper lobe collapse (hypoplasia).

The baby was immediately transferred to the paediatric surgical unit and repair of the defect was done (Figure 2) by the paediatric surgeon. The baby was kept in the intensive care unit post operative for 2 days and discharged in a week. She made an uneventful recovery.

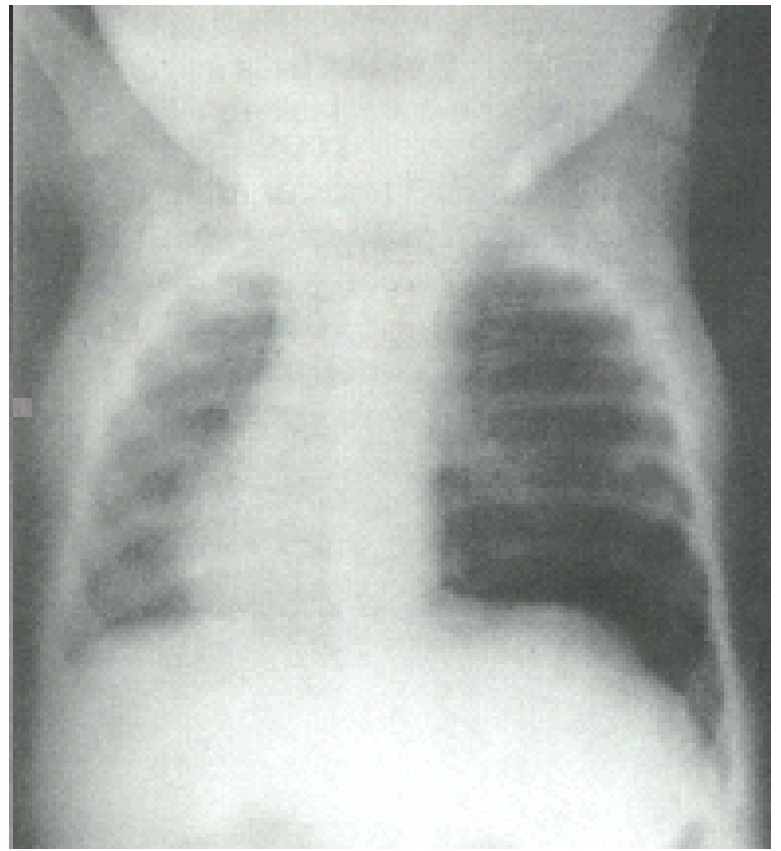

Figure 2. Right sided diaphragmatic hernia (After surgery)

\footnotetext{
${ }^{\mathrm{T}}$ House officer (Paediatric Unit), Durdans Hospital, Colombo

${ }^{2}$ Consultant Paediatrician

${ }^{3}$ Consultant Paediatric Surgeon
} 
